\title{
Squamous cell carcinoma of head and neck: what internists should know
}

\author{
Kyungsuk Jung ${ }^{1}$, Manpreet Narwal ${ }^{2}$, Seon Young Min³, Bhumsuk Keam4, and Hyunseok Kang ${ }^{2}$
}

\begin{abstract}
${ }^{1}$ Department of Medicine, Fox Chase Cancer Center, Philadelphia, PA; Departments of ${ }^{2}$ Medicine and ${ }^{3}$ Oral and Maxillofacial Surgery, University of California San Francisco, San Francisco, CA, USA; ${ }^{4}$ Department of Internal Medicine, Seoul National University Hospital, Seoul, Korea
\end{abstract}

Received: February 29, 2020

Accepted: May 14, 2020

\section{Correspondence to}

Hyunseok Kang, M.D.

Department of Medicine,

University of California San

Francisco, 550 16th Street, San

Francisco, CA 94158, USA

Tel: $+1-4158857356$

Fax: +1-4153337984

E-mail: Hyunseok.kang@ucsf.edu https://orcid.org/o000-00015758-8202
Squamous cell carcinoma of head and neck (SCCHN) is a group of cancer arising from mucosal surfaces of the head and neck. Optimal management of SCCHN requires a multidisciplinary team of surgical oncologists, radiation oncologists, medical oncologists, nutritionist, and speech-language pathologists, due to the complexity of anatomical structure and importance of functional outcome. Human papilloma virus (HPV)-related SCCHN represents a distinct subset from HPV negative SCCHN which is associated with carcinogen exposure such as cigarette smoking, betel nut use and alcohol. HPV related SCCHN responds better to concurrent chemoradiation and has better overall prognosis, compared to HPV negative SCCHN. Radiation therapy has been introduced to the treatment of SCCHN, administered concurrently with systemic chemotherapy for locoregional SCCHN, as well as a palliative measure for recurrent and/or metastatic (R/M) SCCHN. Recently, immune checkpoint inhibitors have been shown to improve overall survival in R/M-SCCHN and have been incorporated into the standard of care. Combination approaches with immune therapy and targeted therapy for biomarker enriched population based on genomics are being actively investigated and will shape the future of SCCHN treatment.

Keywords: Head and neck neoplasms; Papillomaviridae; Survivorship; Drug therapy

\section{INTRODUCTION}

Squamous cell carcinoma of head and neck (SCCHN) comprises a heterogeneous group of epithelial neoplasms that arise from upper aerodigestive tract [1]. Almost 65,000 people are estimated to have been affected by SCCHN in the United States in 2019 [2]. In South Korea, 3,309 new cases of cancer in the lip, oral cavity, and pharynx were diagnosed, and 1,170 patients died from the disease in 2015 [3]. Treatment outcomes of SCCHN patients have improved over the past decades: 5-year survival rate of SCCHN in South Korea had jumped from $41.1 \%$ to $64.5 \%$ in the past 2 decades [3]. This improvement is partly attributed to advance surgical and radiation techniques and better supportive care, but there also has been a major shift in patient characteristics. Historically, smoking and alcohol use were the major etiologic factors of SCCHN with sporadic cases caused by Betel nut chewing or genetic predisposition, such as Fanconi anemia [4-6]. However, over the past decade, human papilloma virus (HPV) has emerged as a pathogen that causes a distinct group of SCCHN, especially oropharyngeal squamous cell carcinoma (SCC) [7]. HPV-related SCCHN affects younger people and has very different genomic features compared to HPV negative SCCHN. After appropriate treatments, HPV-related SCCHN carries significantly better prognosis both in locally advanced disease and in recurrent or metastatic 


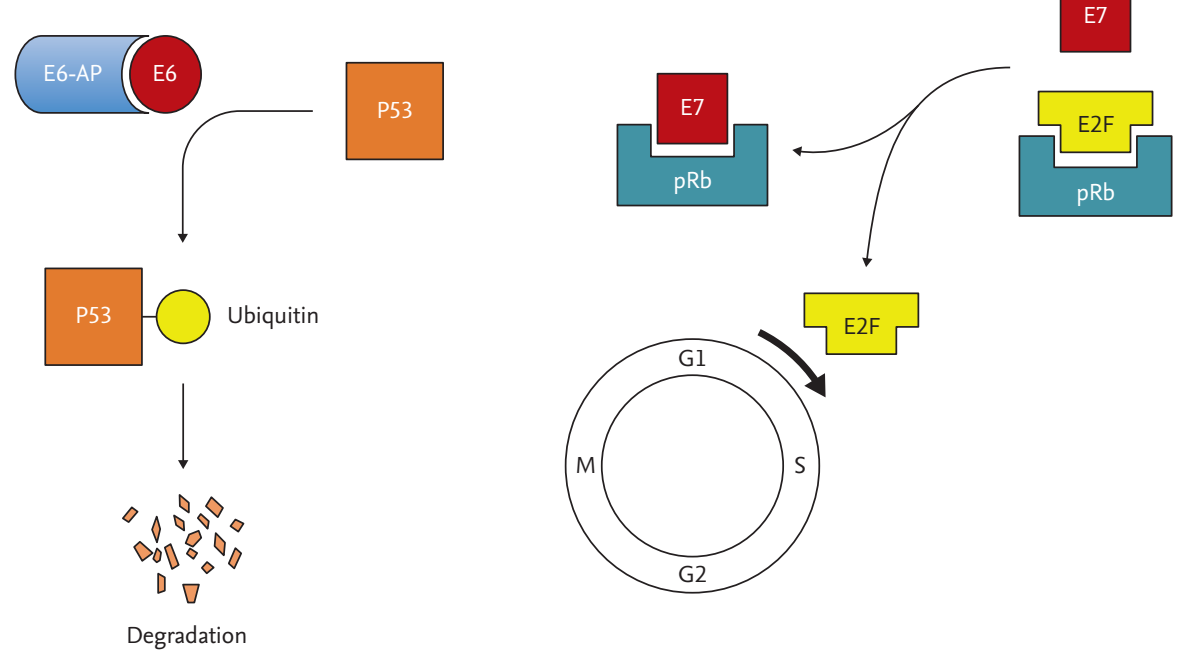

Figure 1. Oncogenic mechanisms of E6 and E7.

disease $[8,9]$. HPV-related SCCHN is predicted to reflect changes in sexual practice, although recent implementation of vaccination program against high risk HPV strains may reverse the trend.

\section{HPV RELATED SCCHN}

\section{Epidemiology}

In 2010, prevalence of oral HPV infection in the United States was estimated to be $6.9 \%$ and HPV16 oral infection was estimated to be $1 \%$ [10]. In another analysis, prevalence of high risk HPV oral infection was estimated to be significantly higher in males than females $(7.3 \%$ vs. $1.4 \%$ ) and males with same-sex partner(s) had even higher infection rate [11]. Among more than $150 \mathrm{HPV}$ serotypes, $\mathrm{HPV} 16$ accounts for the majority of HPV associated SCCHN [12] and it primarily affects oropharynx by infecting lymphoid epithelium [13]. The proportion of HPV-positive disease among oropharyngeal cancer has recently increased over time regardless of sex and race, whereas the overall prevalence of SCCHN has remained stable or slightly decreased. In South Korea, prevalence of HPV varied widely depending on the disease site, ranging from $5.3 \%$ to $14.5 \%$ in oral cavity and from $23.5 \%$ to $73.1 \%$ in oropharynx [14].

Compared to HPV-negative SCCHN, HPV related
SCCHN predominantly affects people with younger age, Caucasian race, and a relatively higher socioeconomic status $[8,15]$. Association with tobacco, alcohol use, and poor dentition is not very strong in HPV related SCCHN. Instead, there is a positive relationship with history of marijuana use [12]. As the viral infection is sexually transmitted, patients with HPV-positive SCCHN tend to have more lifetime sexual partners and more likelihood of having experience of oral-genital or oralanal contact $[15,16]$. In a recent cross-sectional study, prophylactic HPV vaccination has shown to be associated with significantly lower risk of having high risk HPV oral infection [17], which raises hope that population wide $H P V$ vaccination program may end the epidemic of HPV related SCCHN in near future.

\section{Biology}

Once transmitted through close contact, HPV invades into micro-wounds of mucosa, and infects the cells in the basal layer of squamous epithelium. E6 and E7 oncoproteins from early phase of HPV life cycle inactivate two key cell cycle regulators, p53 and pRb [18]. E6 binds with E6-associated protein that has ubiquitin ligase activity, which degrades p53, a key tumor suppressor. On the other hand, E7 competitively binds to $\mathrm{pRb}$, releasing transcription factor E2F, which migrates to nucleus and triggers transcription of several genes involved in 
Table 1. Characteristics of HPV-positive and negative SCCHN

\begin{tabular}{|c|c|c|}
\hline Characteristic & HPV-positive & HPV-negative \\
\hline Incidence & Increasing & Decreasing \\
\hline Disease location & Predominant in oropharynx & Various \\
\hline Age & Younger & Older \\
\hline Race & More prevalent in white & Less prevalent in white \\
\hline Socioeconomic status & Higher & Lower \\
\hline Tobacco/Alcohol & Not strongly associated & Strongly associated \\
\hline Oral hygiene/dentition & Better & Worse \\
\hline Marijuana & Associated & Not associated \\
\hline No. of sexual partners & Higher & Lower \\
\hline Oral sexual contact & More frequent & Less frequent \\
\hline Genomic alterations & $\mathrm{FGFR}_{3}, \mathrm{E}_{2} \mathrm{~F}_{1}, \mathrm{TRAF}_{3}$ & ${ }^{T} P_{53}, C D K N 2 A, M Y C, C C N D_{1}$ \\
\hline Prognosis & Better & Worse \\
\hline
\end{tabular}

HPV, human papilloma virus; SCCHN, squamous cell carcinoma of head and neck; FGFR3, fibroblast growth factor receptor 3; $E_{2} F_{1}, E_{2} F$ transcription factor 1; TRAF3, TNF receptor-associated factor 3; CDKN2A, cyclin-dependent kinase inhibitor 2A; CCND1, cyclin D1.

cell cycle progression and inhibition of apoptosis (Fig. 1) [19]. Both E6 and E7 oncoproteins consequently conduce to abrogation of cell cycle checkpoints, proliferation of cells, and thus, amplification of viral genome. Cells that are persistently infected with HPV continue to propagate, uncoupled from differentiation. These uninhibited cell divisions are prone to accumulated DNA damages and chromosomal abnormalities, which increase oncogenic potential. Although most of the HPV infected cells are removed by immune response in 1 to 2 years, failure to clear the HPV infection results in development of cancer in decades $[20,21]$.

\section{HPV detection for diagnosis}

Release of E2F mediated by E7 increases expression p16, which is used as a surrogate biomarker for HPV related oropharyngeal cancer (Fig. 2) [22]. Immunohistochemistry (IHC) for p16 in oropharyngeal SCCs have high sensitivity and specificity and can be reliably used to diagnose HPV related SCCHN [23]. For non-oropharyngeal SCCs, prevalence of HPV related SCCHN is much lower which makes p16 IHC unreliable. HPV DNA detection by polymerase chain reaction has been regarded as the gold standard, but recently direct visualization using RNA probe (RNA in situ hybridization) has been shown to be highly sensitive and specific in detecting transcriptionally active HPV in oropharyngeal cancers [24].

\section{Genomics}

Comprehensive genomic analysis of SCCHN in The Cancer Genome Atlas (TCGA) demonstrated significant contrasts in the distribution of genomic alterations between HPV-positive and negative SCCHN [25]. HPV-positive SCCHN harbored frequent alterations of $\mathrm{PIK}_{3} \mathrm{CA}$ and TNF receptor-associated factor 3 (TRAF3), whereas mutations or copy number variations of TP53, cyclin-dependent kinase inhibitor 2A (CDKN2A), MYC, and cyclin D1 (CCND1) predominantly affected HPV-negative SCCHN. TRAF 3 , frequently affected in HPV-positive diseases, plays a critical role in innate immune defense, and cells lacking TRAF3 has impaired interferon response against viral infection [26]. Focal amplifications of receptor tyrosine kinase, such as EGFR, $E R B B 2$, and fibroblast growth factor receptor 1 (FGFR1), favored HPV-negative disease. The most common genomic alteration in HPV-positive SCCHN was a helical domain mutation of $\mathrm{PIK}_{3} \mathrm{CA}$ which encodes a subunit of class 1 phosphoinositide 3-kinase ( $\left.\mathrm{PI}_{3} \mathrm{~K}\right)$ [27]. $\mathrm{PI}_{3} \mathrm{~K}$ receives a signal from cell surface receptors, such as EGFR, and attaches a phosphate group to the inositol head of phosphatidylinositol 4,5-bisphosphate (PIP2) in the cell membrane, converting it to phosphatidylinositol 3,4,5-triphosphate ( $\mathrm{PIP}_{3}$ ) [28]. PIP3, in turn, sends down the signal of cellular growth through the cascade of Akt/ protein kinase $\mathrm{B}$ and mammalian target of rapamycin 
(mTOR) [29]. In the TCGA data, mutations in the $\mathrm{PI}_{3} \mathrm{~K}-$ CA were concentrated in the frequent 'hotspot' mutations ( $\mathrm{E}_{542}, \mathrm{E}_{545}$ in the helical domains) in HPV-positive SCCHN, whereas mutations were more spread out in HPV-negative diseases $[25,27,30,31]$.

\section{Clinical features}

Patients with HPV-positive SCCHN tend to have less smaller primary tumors, larger cystic lymph node, better performance status, and better prognosis compared to patients with HPV negative SCCHN [8,32]. In vitro studies also have shown that HPV infected cancer cells are more prone to apoptosis in response to DNA damaging agents [33,34]. In a prospective cohort study of oropharyngeal and laryngeal SCC, patients with HPV-positive disease had longer progression free survival (PFS) and overall survival(OS), and the improved survival persisted after careful adjustment for age, tumor stage, and Eastern Cooperative Oncology Group (ECOG) performance status. Considering the sensitivity to treatment and better prognosis, patients with HPV-positive SCCHN may benefit from less toxic, de-intensified treatment strategy, which should be a focus of future studies. Differences of disease characteristics between HPV-positive and negative SCCHN are summarized in Table 1.

\section{CURRENT STANDARD OF TREATMENT}

\section{Locally advanced SCCHN}

About $40 \%$ of the patients with SCCHN initially present with early stage, localized disease [35]. These patients can be effectively treated with a single modality therapy, either curative intent surgery or definitive radiation, depending on tumor location and the institution's experience. While surgery has been the traditional treatment for these cancers, definitive radiation can safely replace surgery with comparable oncologic outcomes, and achieve better functional outcomes in larynx, hypopharynx, or oropharynx.

For patients with locally advanced SCCHN, multi-modality therapy involving at least two different modalities are required. If upfront surgery is offered, post-operative (adjuvant) radiation therapy should be considered with or without concurrent chemotherapy. Definitive radiotherapy with concurrent chemotherapy is also widely used, but it is not preferred upfront approach for oral cavity SCCHN for inferior oncologic outcomes and higher complication rates [36]. Recent developments in minimally invasive surgery, such as transoral robotic surgery (TORS), has enabled surgeons to gain easier access to certain areas of oropharynx such as base of tongue, which traditionally required an extensive procedure involving split of mandible. TORS has been widely adopted for treatment of HPV positive oropharyngeal SCC, as patients are expected liver longer and more motivated to avoid late consequences from radiotherapy and/or chemotherapy. A small randomized phase 2 trial comparing upfront TORS versus definitive (chemo)radiotherapy demonstrated that upfront radiotherapy was slightly better in terms of swallowing related quality of life (QOL) measure, but the difference was not clinically meaningful [37]. More clinical trials comparing definitive radiation and TORS are on-going in early stage oropharyngeal SCCs.

Chemotherapy alters DNA binding and creates reactive free radicals, enhancing the cytotoxic effect of radiation [38]. Besides, chemotherapy inhibits DNA repair and recovery from potentially lethal or sub-lethal damages in between radiation sessions [39]. Several clinical trials have confirmed that concomitant administration of chemoradiation significantly prolongs survival for locally advanced SCCHN, compared to radiation alone, or sequential administration of chemotherapy and radiation [40-45]. Concurrent chemoradiation was superior to radiation alone in postoperative settings as well for patients who have high risk pathologic features such as positive margins and/or extranodal extension $[46,47]$. Based on these findings, concurrent chemoradiation has been widely adopted for the treatment of locally advanced SCCHN.

Induction chemotherapy prior to definitive radiation or chemoradiation has been contemplated for long time to achieve immediate response and decrease risk of distant metastasis. However, a series of clinical trials of induction chemotherapy compared to upfront concurrent chemoradiation failed to demonstrate superior survival, even in the patients with high risk patients with bulky tumor or lymph node [48-50]. Furthermore, toxicities from induction chemotherapy may delay or interrupt definitive chemoradiation. Neoadjuvant chemotherapy prior to definitive surgery has been evaluated in a large 
phase 3 randomized trial in oral cavity cancers, but failed to demonstrate any overall or disease-free survival benefit [51]. Therefore, induction or neoadjuvant chemotherapy is not considered a standard treatment for locally advanced SCCHN. Nonetheless, for patients with bulky, symptomatic primary or nodal disease, induction chemotherapy can be started if the initiation of chemoradiation is delayed.

Cetuximab, an anti-EGFR antibody, has been used as a concurrent therapy with radiation. Cetuximab plus radiation demonstrated OS and PFS benefits compared to radiation alone for patients with locally advanced SCCHN [52], and cetuximab was viewed as a way to decrease treatment-related toxicity in definitive concurrent chemoradiation, especially for HPV related SCCHN patients who have excellent prognosis. Two large randomized clinical trials evaluated concurrent cetuximab in comparison to concurrent cisplatin with definitive radiotherapy in locoregionally advanced SCCHN with intent of demonstrating non-inferiority of outcomes. However, to the contrary of the expectations, concurrent cetuximab with radiation was shown to have worse OS and PFS in both studies [53,54]. For HPV negative SCCHN, it is not clear whether cisplatin would be still superior to cetuximab as a concurrent treatment, but cisplatin remains to be the standard concurrent systemic agent.

\section{Recurrent and/or metastatic SCCHN}

Although majority of patients with SCCHN present with locally advanced disease, approximately $30 \%$ to $40 \%$ of patients develop recurrence even with intensive multimodality treatment [45]. For selected patients with locoregional recurrence, surgical salvage or re-irradiation can be attempted, but it has not been very successful [55]. Recent retrospective case series suggests that surgical salvage for locoregional and distant failure in oropharyngeal SCC can be associated with improved OS in both HPV-positive and -negative patients, although the benefit in oligometastatic disease was largely limited to HPV positive disease [56]. Even after recurrent or metastatic disease, patients with HPV positive SCCHN have longer OS compared to the ones with HPV negative SCCHN $[9,57]$. However, median disease free survival after salvage surgery remains poor for both HPV-positive and HPV-negative patients [58]. Metastatic and/or recurrent disease not amenable for surgical salvage can be treated with systemic therapy which includes cytotoxic chemotherapy, targeted therapy and immune checkpoint inhibitors.

Combination of platinum, fluorouracil, and cetuximab (EXTREME regimen), which was shown to prolong OS to 10.1 months, used to be the standard of care for the 1st line treatment of recurrent and/or metastatic SCCHN [59]. Cetuximab has a modest single agent activity for SCCHN when used after failing prior platinum-based chemotherapy [6o]. Generally, conventional systemic treatment options are limited as responses are generally short-lived and not durable with high toxicity toll. Immune checkpoint inhibitors bind to molecules that mediates immune tolerance such as programmed death 1 (PD1) receptors which are located on the membrane of T cells, B cells, and natural killer cells. When bound with ligands on tumor cell surface, these immune checkpoint molecules inhibit cancer cell apoptosis, and down-regulate cytotoxic $\mathrm{T}$ cell function $[61,62]$. Immune checkpoint inhibitors re-stimulate the immune function to target cancer cells.

Anti-PD1 antibodies have been evaluated in recurrent/ metastatic (RM)-SCCHN patients in the 2nd line setting after progression on platinum containing chemotherapy. Pembrolizumab demonstrated overall response rate of $16 \%$ in a single arm study, which led to accelerated approval of pembrolizumab by the U.S. Food and Drug Administration. This trial included even patients with no programmed death-ligand 1 (PD-L1) expression, and $12 \%$ of these patients still demonstrated objective responses. Response rates were comparable between HPV-positive and negative groups [63]. Nivolumab was evaluated in a phase 3 randomized clinical trial, compared to a single agent therapy of investigators' choice. This study demonstrated significant OS benefit with 1-year survival rate of $36 \%$ versus $16.6 \%$ [64]. Of note, patients who received nivolumab had better QOL outcome in terms of social function, fatigue, dyspnea, pain, and appetite. Median time to deterioration in several domains of QOL was significantly longer with nivolum$\mathrm{ab}$ than with standard chemotherapy [65]. Nivolumab is currently approved by the U.S. Food and Drug Administration for the treatment of recurrent or metastatic SCCHN after progression on platinum-based chemotherapy.

More recently, pembrolizumab with or without che- 
motherapy was evaluated in the 1st line RM-SCCHN in comparison to the EXTREME regimen in a randomized, phase 3 clinical trial [66]. The study demonstrated that pembrolizumab alone has significant OS benefit in patients with PD-L1 expressing tumor (defined by combined positive score $>1$ ) and combination of pembrolizumab and chemotherapy has significant OS benefit in all patients. Pembrolizumab with or without chemotherapy was approved based on this study for the ist line use in RM-SCCHN. Of note, overall response rate of pembrolizumab alone was significantly lower than conventional chemotherapy, while that of pembrolizumab plus chemotherapy was comparable to the chemotherapy. This implies that pembrolizumab and chemotherapy combination might be more beneficial for or patients with bulky disease who would benefit from rapid reduction of tumor burden, regardless of PD-Li expression status.

Approval of immune checkpoint inhibitors in the 1st line treatment of RM-SCCHN limits options for and line treatment. A study suggests chemotherapy would be still active and viable option for patients who received immune checkpoint inhibitors in the first line setting. Objective response was seen in $37.5 \%$ of 16 patients who received cetuximab after anti-PD1 failure and $44.4 \%$ of 27 patients who received chemotherapy based regimen [67].

\section{MULTIDISCIPLINARY CARE AND IMPORTANCE OF FUNCTIONAL OUTCOME}

SCCHN involves very complex and delicate structures. Mucosal surface of oral cavity, pharynx and larynx covers vital passage of food and air and located very close to other important anatomical areas such as skull base and carotid artery. Oral cavity, pharynx and larynx are responsible for normal breathing, swallowing and speech. Treatment of SCCHN may impact all of these functions and may cause trismus, neck and shoulder dysfunction, vascular complications, dysphagia, xerostomia, dental caries, taste disorder, fatigue, sleep dysfunction, and hypothyroidism [68]. These long-term consequences of treatment can lead to loss of appetite, weight loss and chronic aspiration induced lung damage and feeding tube dependency, which negatively impact patients' QOL and body image. Long term consequences from treatment of head and neck cancer can vary depending on treatment modality used, thus it is important to consider functional outcome in addition to the oncological outcome when making a treatment decision.

It is important to remember that concurrent chemoradiation was developed as a way to preserve larynx function in patients with laryngeal SCC. Radiation Therapy Oncology Group (RTOG) 91-11 study, which randomized stage III/IV laryngeal cancer patients to the Veterans Affairs (VA) laryngeal study approach (induction chemotherapy then radiation), concurrent chemoradiation (cisplatin), and radiation alone, showed that concurrent chemoradiation was superior to the others in achieving laryngeal preservation and locoregional control [43]. However, there was a substantial risk of overall treatment failure with $25 \%$ of the entire study population eventually receiving salvage laryngectomy. Moreover, patients with a high volume $\mathrm{T}_{4}$ disease were excluded in this study leaving $90 \%$ of the participants with $\mathrm{T}_{2}$ or $\mathrm{T}_{3}$ tumors. In patients with bulky diseases treated with non-surgical approach, the clinical benefit of sparing already deformed larynx is possibly low because of subsequent aspiration risk [69]. It was also noted in a long-term follow-up that there was a possibility of worse outcome from concurrent chemoradiation as more deaths occurred from causes other than laryngeal cancer [45].

Radiation treatment is the frequent culprit for pharyngeal constrictor muscle damage and intractable aspiration [70]. It has been demonstrated that there is a positive relationship between radiation dose and volume/thickness of pharyngeal constrictor muscle as well as level of acoustic-articular changes in patients with oropharyngeal cancer $[71,72]$. Toxicities from radiation or chemotherapy often exacerbate voice or swallowing function in an already dysfunctional, cancer-riddled upper aerodigestive tract. Among the patients with oropharyngeal cancer who had been treated with radiation with or without chemotherapy, Penetration Aspiration Scale (PAS) was abnormal in $45 \%$ of them, which was also independently associated with pre-treatment swallowing difficulty [73]. Nonetheless, patients with SCCHN have demonstrated coping mechanisms and self-adjustment. In an assessment of voice and swallowing function using the Voice Related Quality of Life (VR-QOL) measure and the list Performance Status Scale for Head 


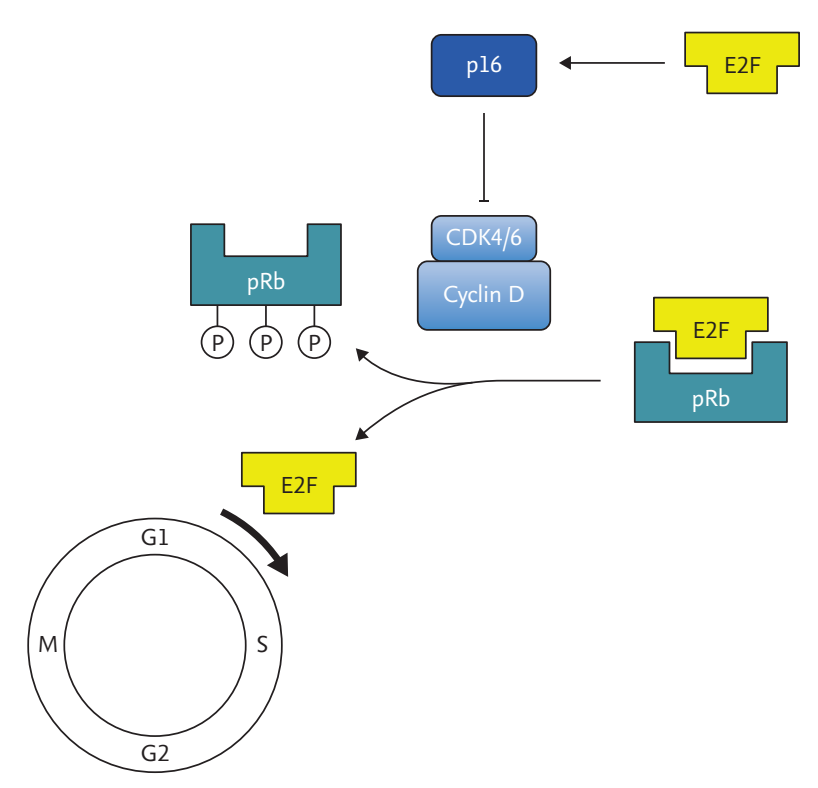

Figure 2. Inhibitory action of p16 on cyclin-dependent kinase (CDK) $4 / 6$-cyclin D complex and cell cycle.

and Neck Cancer Patients (PSS-HN), patients with laryngeal cancer who had received organ preservation treatment of concurrent chemoradiation, although, understandably, scored worse than normal subjects, had higher QOL scores than patients with non-cancerous voice disorders, such as vocal fold paralysis or adductor spasmodic dysphonia. Their QOL results were also significantly higher than patients who underwent laryngectomy, especially in terms of understandability of speech. However, swallowing function, dietary restriction, and frequency of eating in public was not better compared to those who had laryngectomy. Factors that were associated with likelihood of eating in public were, instead, longer time duration since the last treatment and lower grade of mucositis [74].

Interestingly, patient's perception of QOL does not seem to correlate with objectively measured level of swallow dysfunction [75], which provides an important clue in understanding patients' disease awareness and perception of QOL. Self-reported symptoms by patients should not be solely relied upon when assessing swallowing capacity and nutritional status as they are not accurate indicators of level of morbidity.

With regard to swallowing dysfunction, patients may need a gastric feeding tube for nutritional support during chemoradiation. Feeding tubes can be placed prophylactically prior to initiation of therapy or reactively when patients develops need for nutritional support. A systematic review suggests that prophylactic feeding tube reduces the number of malnourished (> 10\% loss of body weight) patients and improves QOL after treatment, but does increase chance for long term feeding tube dependence [76]. A retrospective review of treatment outcome of concurrent platinum-based chemoradiation for advanced laryngeal cancer showed that $7 \%$ of the patients required percutaneous gastrostomy, $6 \%$ had persistent dysphagia, and $2 \%$ had chronic lung aspiration [77].

Salivary gland is sensitive to radiation and can be easily damaged by radiation causing permanent dysfunction [78]. Xerostomia and hyposalivation are common late toxicities of head and neck radiotherapy and can contribute to the development of dental demineralization and caries. Approximately $21 \%$ to $24 \%$ of SCCHN patients treated with radiation or chemoradiation develops dental caries [79], due to decreased buffering capacity, insufficient calcium and phosphate, proliferation of cariogenic bacteria and diet change [8o]. Dental demineralization may lead to rampant dental breakdown and osteonecrosis. Therefore, a comprehensive dental evaluation is essential, and any underlying dental problems associated with poor dental outcome need to be addressed prior to initiation of radiotherapy. Ongoing dental care including maintenance of good oral hygiene and regular supplementation of fluorides is necessary after therapy [81].

\section{SURVIVORSHIP AND SUPPORTIVE CARE}

Patients with SCCHN are living longer as novel pharmaceuticals and therapeutic techniques are being developed. Among the patients with HPV-positive disease, 5-year survival rate reached nearly 90\% [82]. Patients who previously underwent invasive treatment for SCCHN frequently suffer from long-term functional or esthetic sequelae and bear a concern or fear of cancer recurrence in their daily lives [83]. As a result, they are more vulnerable to psychosocial illnesses, such as depression and anxiety, which negatively impact social and occupational activities. Therefore, SCCHN patients often require close and personalized psychosocial care 


\section{KJIM}

Table 2. Key recommendations for supportive treatment and survivorship care for SCCHN

\begin{tabular}{|c|c|}
\hline \multicolumn{2}{|l|}{ Pre-treatment evaluation } \\
\hline Multidisciplinary approach & $\begin{array}{l}\text { Assessment and evaluation by multiple subspecialties of oncology } \\
\text { Possible treatment de-intensification or organ preservation for HPV-positive or locoregional } \\
\text { disease } \\
\text { Consider reconstruction if extensive surgical resection is required }\end{array}$ \\
\hline Speech/language therapy & $\begin{array}{l}\text { Voice therapy (use electrolarynx if necessary) } \\
\text { Swallow exercise } \\
\text { Close follow-up and objective assessment of speech and swallowing function (patient-reported } \\
\text { symptoms do not accurately reflect the level of morbidity) }\end{array}$ \\
\hline Nutrition & $\begin{array}{l}\text { Prophylactic or elective G tube feeding } \\
\text { Dental and oral care }\end{array}$ \\
\hline \multicolumn{2}{|l|}{ Survivorship care } \\
\hline Cancer surveillance & $\begin{array}{l}\text { Cancer-specific history and physical exam every } 1 \text { to } 3 \text { months for the first year after the } \\
\text { primary treatment, every } 2 \text { to } 6 \text { months in the second year, every } 4 \text { to } 8 \text { months in the } 3 \text { rd to } \\
\text { 5th years, and annually after the 5th year } \\
\text { Patient education about the signs of early recurrence } \\
\text { Adequate and timely referral to oncologist or ENT specialist if detailed exam is needed } \\
\text { Adhere to national guidelines for second primary cancer prevention }\end{array}$ \\
\hline Substance abuse & $\begin{array}{l}\text { Proper counseling on smoking cessation and alcohol abstinence } \\
\text { Screen for underlying mood disorder } \\
\text { Referral to cessation resources or psychiatrist if needed }\end{array}$ \\
\hline Body and self-image & $\begin{array}{l}\text { Assessment of body and self-image concerns } \\
\text { Referral to psychiatrist if needed }\end{array}$ \\
\hline Mood disorder & $\begin{array}{l}\text { Evaluate survivors for depression, anxiety and screen for relevant social/financial/emotional } \\
\text { distresses } \\
\text { Social care for financial or employment challenges } \\
\text { Addiction recovery assistance for substance abuse } \\
\text { Pharmacologic treatment as indicated } \\
\text { Timely referral to psychiatrist }\end{array}$ \\
\hline
\end{tabular}

SCCHN, squamous cell carcinoma of head and neck; HPV, human papilloma virus; ENT, ear nose throat.

for a long-term period after the treatment. Comprehensive care for SCCHN survivors also includes assessment and management of late-occurring symptoms, general health promotion, and surveillance of recurrence or second primary cancer. To meet each individual's needs and requirements, SCCHN survivorship program necessitates multidisciplinary efforts from primary care, speech therapy, physical therapy, social care, psychiatry and oncology subspecialties.

American Cancer Society (ACS) Head and Neck Cancer Survivorship Care Guideline recommends that primary care physician should obtain cancer-related history and physical exam every 1 to 3 months for the 1st year after the primary treatment, every 2 to 6 months in the 2nd year, every 4 to 8 months in the 3 rd to 5 th years, and annually after the 5 th year. Patients should continue follow-up with head and neck surgeons for more detailed and focused exam. Primary care physicians are also responsible for educating survivors about the signs of early recurrence. For early detection of second primary cancer, patients should adhere to national guidelines for general cancer prevention [84].

Smoking and alcohol abuse, which is common among patients with SCCHN, can increase the risk of second primary cancer. However, smoking and alcohol cessation rates after the cancer diagnosis are inadequately low. Among the SCCHN patients with previous or current smoking history at the time of diagnosis, approximately $25 \%$ to $35 \%$ of them continued to smoke after the treatment $[85,86]$, and the smoking rate was higher 
after non-surgical treatment compared to surgery [85]. Furthermore, alcohol-dependent behavior became increased 1 year after the SCCHN diagnosis regardless of treatments received [87]. Therefore, it is important to counsel the survivors on smoking cessation and alcohol use.

SCCHN survivors frequently experience dramatic changes in self-perception of body image. This concerns multiple aspects of psychosocial outcome, causing mood disorders and avoidance of social activity. Their social function is more impaired when they feel discouraged to eat or speak in public due to self-consciousness of facial difference. Severe changes in self-perception may also jeopardize employment and financial status [88]. These issues may contribute to high prevalence of mood disorders among SCCHN survivors: $17 \%, 15 \%$, and $13 \%$ of SCCHN patients who were disease free after radiation treatment reported that they are "somewhat depressed" or "extremely depressed" after 1, 3, and 5 years, respectively. In spite of high prevalence of depression at 5 years, none of the patients were receiving anti-depressants or psychotherapy at the time [89]. Direct comparison should be interpreted with caution, but prevalence of depression was $11.6 \%$ among the cancer survivors (>2 years) in general and $10.2 \%$ in healthy controls [90]. Managing mood disorder for SCCHN survivors is crucial as depression is negatively correlated with OS and disease recurrence, even after adjusting for other relevant factors [91]. It is incumbent on primary care physicians to evaluate SCCHN survivors for depression or anxiety every three months post-treatment using an appropriate diagnostic tool. Patients should be offered pharmacologic interventions or referral to specialists if indicated. Comprehensive management of mental distress also includes social care for financial and employment challenges, or addiction recovery assistance for substance abuse. Key recommendations for supportive treatment and survivorship care are summarized in Table 2.

\section{CONCLUSIONS}

Current researches on head and neck cancer treatment are focused on a few areas. For locoregionally advanced SCCHN, there is an effort to reduce the burden of treatment related toxicities with equivalent outcomes for
HPV related disease. The efforts include adoption of induction chemotherapy as a selection tool for patients to receive reduced amount of radiation and use of reduced amount radiation in combination with immune checkpoint inhibitors or chemotherapy. ECOG 1380 study was a phase 2 study which used induction chemotherapy upfront for HPV positive SCCHN. If patients respond to the chemotherapy, they received reduced amount of radiation (54 Gy as opposed to $70 \mathrm{~Gy}$ ) with concurrent cetuximab. 70\% of patients received reduced amount of radiation and their 2-year PFS was 96\% [92]. This study suggests that induction chemotherapy can be used as a patient selection tool for treatment de-intensification. For HPV positive disease, NRG HNoo2 study was a phase 2 study with reduced dose radiation (6o Gy) with or without concurrent cisplatin for favorable risk HPV positive SCCHN. The radiation alone arm did not meet the prespecified 2 year PFS goal of $85 \%$, while the concurrent cisplatin arm did meet the goal [93]. Based on the result, NRG HNoo5 study is being conducted to compare standard of care (7o Gy plus cisplatin), reduced dose radiation with cisplatin and reduced dose radiation with an immune checkpoint inhibitor, nivolum$\mathrm{ab}$ (NCT03952585). For HPV negative, locoregionally advanced SCCHN, efforts are focused on intensifying current standard of care to improve the suboptimal outcome. Many clinical trials are investigating addition of immune checkpoint inhibitor to the concurrent chemoradiation (NCT03040999, NCTo2952586) or after the concurrent chemoradiation (NCTo3452137).

For RM-SCCHN, many researches are focused on enhancing the activity of immune checkpoint inhibitors by adding more agents including HPV vaccine, immune adjuvants, or other immune checkpoint inhibitors. For example, an HPV vaccine, ISA101, demonstrated objective response rate (ORR) of $33 \%$ in combination with nivolumab in a phase 2 study [94]. However, these activities need to be interpreted with caution and should be confirmed in larger phase 3 studies and interpreted with caution. Recently, precision oncology-based approaches are gaining more attention in RM-SCCHN. Traditionally, frequent mutations in SCCHN were thought to be non-actionable as most of the genomic alterations were found in tumor suppressor genes such as TP53. However, a small number of SCCHN does harbor distinct oncogenic driver mutations in genes like 
HRAS and PIK 3 CA. A recent clinical trial with tipifarnib, a farnesyl-transferase inhibitor which inhibits activity of HRAS, demonstrated 56\% ORR in 18 SCCHN patients with HRAS mutations [95], and a larger study is underway to confirm the activity.

There are also research efforts to reduce the toll of toxicities related to treatment. Superoxide dismutase mimetics have been thought to be agents to alleviate radiation related toxicities. A randomized, phase 2 study of GC4419, a superoxide dismutase mimetic, versus placebo, in locoregionally advanced SCCHN, demonstrated that GC4419 does decrease incidence of several oral mucositis [96]. Agents in this class are being under evaluation in larger studies at this time.

\section{Conflict of interest}

No potential conflict of interest relevant to this article was reported.

\section{REFERENCES}

1. Walden MJ, Aygun N. Head and neck cancer. Semin Roentgenol 2013;48:75-86.

2. Siegel RL, Miller KD, Jemal A. Cancer statistics, 2019. CA Cancer J Clin 2019;69:7-34.

3. Jung KW, Won YJ, Kong HJ, Lee ES; Community of Population-Based Regional Cancer Registries. Cancer statistics in Korea: incidence, mortality, survival, and prevalence in 2015. Cancer Res Treat 2018;50:303-316.

4. Goldenberg D, Lee J, Koch WM, et al. Habitual risk factors for head and neck cancer. Otolaryngol Head Neck Surg 2004;131:986-993.

5. Maier H, Dietz A, Gewelke U, Heller WD, Weidauer H. Tobacco and alcohol and the risk of head and neck cancer. Clin Investig 1992;70:320-327.

6. Kutler DI, Auerbach AD, Satagopan J, et al. High incidence of head and neck squamous cell carcinoma in patients with Fanconi anemia. Arch Otolaryngol Head Neck Surg 2003;129:106-112.

7. D'Souza G, Kreimer AR, Viscidi R, et al. Case-control study of human papillomavirus and oropharyngeal cancer. N Engl J Med 2007;356:1944-1956.

8. Ang KK, Harris J, Wheeler R, et al. Human papillomavirus and survival of patients with oropharyngeal cancer. $\mathrm{N}$ Engl J Med 2010;363:24-35.
9. Fakhry C, Zhang Q, Nguyen-Tan PF, et al. Human papillomavirus and overall survival after progression of oropharyngeal squamous cell carcinoma. J Clin Oncol 2014;32:3365-73.

10. Gillison ML, Broutian T, Pickard RK, et al. Prevalence of oral HPV infection in the United States, 2009-2010. JAMA 2012;307:693-703.

11. Sonawane K, Suk R, Chiao EY, et al. Oral human papillomavirus infection: differences in prevalence between sexes and concordance with genital human papillomavirus infection, NHANES 2011 to 2014. Ann Intern Med 2017;167:714-724.

12. Gillison ML, D'Souza G, Westra W, et al. Distinct risk factor profiles for human papillomavirus type 16-positive and human papillomavirus type 16-negative head and neck cancers. J Natl Cancer Inst 2008;100:407-420.

13. Mork J, Lie AK, Glattre E, et al. Human papillomavirus infection as a risk factor for squamous-cell carcinoma of the head and neck. N Engl J Med 2001;344:1125-1131.

14. Kim YT, Serrano B, Lee JK, et al. Burden of human papillomavirus (HPV)-related disease and potential impact of HPV vaccines in the Republic of Korea. Papillomavirus Res 2019;7:26-42.

15. Smith EM, Ritchie JM, Summersgill KF, et al. Age, sexual behavior and human papillomavirus infection in oral cavity and oropharyngeal cancers. Int J Cancer 2004;108:766-772.

16. Anaya-Saavedra G, Ramirez-Amador V, Irigoyen-Camacho ME, et al. High association of human papillomavirus infection with oral cancer: a case-control study. Arch Med Res 2008;39:189-197.

17. Chaturvedi AK, Graubard BI, Broutian T, et al. Effect of prophylactic human papillomavirus (HPV) vaccination on oral HPV infections among young adults in the United States. J Clin Oncol 2018;36:262-267.

18. Mittal S, Banks L. Molecular mechanisms underlying human papillomavirus E6 and E7 oncoprotein-induced cell transformation. Mutat Res Rev Mutat Res 2017;772:23-35.

19. Ertosun MG, Hapil FZ, Osman Nidai O. E2F1 transcription factor and its impact on growth factor and cytokine signaling. Cytokine Growth Factor Rev 2016;31:17-25.

20. Ho GY, Bierman R, Beardsley L, Chang CJ, Burk RD. Natural history of cervicovaginal papillomavirus infection in young women. N Engl J Med 1998;338:423-428.

21. zur Hausen H. Papillomaviruses causing cancer: evasion from host-cell control in early events in carcinogenesis. J 
Natl Cancer Inst 2000;92:690-698.

22. McLaughlin-Drubin ME, Crum CP, Munger K. Human papillomavirus E7 oncoprotein induces KDM6A and KDM6B histone demethylase expression and causes epigenetic reprogramming. Proc Natl Acad Sci U S A 2011;108:2130-2135.

23. Prigge ES, Arbyn M, von Knebel Doeberitz M, Reuschenbach M. Diagnostic accuracy of p16INK4a immunohistochemistry in oropharyngeal squamous cell carcinomas: a systematic review and meta-analysis. Int J Cancer 2017;140:1186-1198.

24. Randen-Brady R, Carpen T, Jouhi L, et al. In situ hybridization for high-risk HPV E6/E7 mRNA is a superior method for detecting transcriptionally active HPV in oropharyngeal cancer. Hum Pathol 2019;90:97-105.

25. Cancer Genome Atlas Network. Comprehensive genomic characterization of head and neck squamous cell carcinomas. Nature 2015;517:576-582.

26. Oganesyan G, Saha SK, Guo B, et al. Critical role of TRAF3 in the Toll-like receptor-dependent and -independent antiviral response. Nature 2006;439:208-211.

27. Koncar RF, Feldman R, Bahassi EM, Hashemi Sadraei N. Comparative molecular profiling of HPV-induced squamous cell carcinomas. Cancer Med 2017;6:1673-1685.

28. Lee JY, Engelman JA, Cantley LC. Biochemistry. $\mathrm{PI}_{3} \mathrm{~K}$ charges ahead. Science 2007;317:206-207.

29. Scheid MP, Woodgett JR. PKB/AKT: functional insights from genetic models. Nat Rev Mol Cell Biol 2001;2:760768.

30. Nichols AC, Palma DA, Chow W, et al. High frequency of activating $\mathrm{PIK}_{3} \mathrm{CA}$ mutations in human papillomavirus-positive oropharyngeal cancer. JAMA Otolaryngol Head Neck Surg 2013;139:617-622.

31. Jung K, Kang H, Mehra R. Targeting phosphoinositide 3-kinase $\left(\mathrm{PI}_{3} \mathrm{~K}\right)$ in head and neck squamous cell carcinoma (HNSCC). Cancers Head Neck 2018;3:3.

32. Chung $\mathrm{CH}$, Zhang Q, Kong CS, et al. p16 protein expression and human papillomavirus status as prognostic biomarkers of nonoropharyngeal head and neck squamous cell carcinoma. J Clin Oncol 2014;32:3930-3938.

33. Butz K, Geisen C, Ullmann A, Spitkovsky D, Hoppe-Seyler F. Cellular responses of HPV-positive cancer cells to genotoxic anti-cancer agents: repression of E6/E7-oncogene expression and induction of apoptosis. Int J Cancer 1996;68:506-513.

34. Liu Y, McKalip A, Herman B. Human papillomavirus type
16 E6 and HPV-16 E6/E7 sensitize human keratinocytes to apoptosis induced by chemotherapeutic agents: roles of p53 and caspase activation. J Cell Biochem 2000;78:334349.

35. Marur S, Forastiere AA. Head and neck squamous cell carcinoma: update on epidemiology, diagnosis, and treatment. Mayo Clin Proc 2016;91:386-396.

36. Zhang H, Dziegielewski PT, Biron VL, et al. Survival outcomes of patients with advanced oral cavity squamous cell carcinoma treated with multimodal therapy: a multi-institutional analysis. J Otolaryngol Head Neck Surg 2013;42:30.

37. Nichols AC, Theurer J, Prisman E, et al. Radiotherapy versus transoral robotic surgery and neck dissection for oropharyngeal squamous cell carcinoma (ORATOR): an open-label, phase 2, randomised trial. Lancet Oncol 2019;20:1349-1359.

38. Richmond RC, Powers EL. Radiation sensitization of bacterial spores by cis-dichlorodiammineplatinum(II). Radiat Res 1976;68:251-257.

39. Wallner KE, Li GC. Effect of cisplatin resistance on cellular radiation response. Int J Radiat Oncol Biol Phys 1987;13:587-591.

40. Calais G, Alfonsi M, Bardet E, et al. Randomized trial of radiation therapy versus concomitant chemotherapy and radiation therapy for advanced-stage oropharynx carcinoma. J Natl Cancer Inst 1999;91:2081-2086.

41. Denis F, Garaud P, Bardet E, et al. Final results of the 94o1 French Head and Neck Oncology and Radiotherapy Group randomized trial comparing radiotherapy alone with concomitant radiochemotherapy in advanced-stage oropharynx carcinoma. J Clin Oncol 2004;22:69-76.

42. Adelstein DJ, Li Y, Adams GL, et al. An intergroup phase III comparison of standard radiation therapy and two schedules of concurrent chemoradiotherapy in patients with unresectable squamous cell head and neck cancer. J Clin Oncol 2003;21:92-98.

43. Forastiere AA, Goepfert H, Maor M, et al. Concurrent chemotherapy and radiotherapy for organ preservation in advanced laryngeal cancer. N Engl J Med 2003;349:20912098.

44. Wendt TG, Grabenbauer GG, Rodel CM, et al. Simultaneous radiochemotherapy versus radiotherapy alone in advanced head and neck cancer: a randomized multicenter study. J Clin Oncol 1998;16:1318-1324.

45. Forastiere AA, Zhang Q, Weber RS, et al. Long-term 
results of RTOG 91-11: a comparison of three nonsurgical treatment strategies to preserve the larynx in patients with locally advanced larynx cancer. J Clin Oncol 2013;31:845-852.

46. Bernier J, Domenge C, Ozsahin M, et al. Postoperative irradiation with or without concomitant chemotherapy for locally advanced head and neck cancer. N Engl J Med 2004;350:1945-1952.

47. Cooper JS, Pajak TF, Forastiere AA, et al. Postoperative concurrent radiotherapy and chemotherapy for high-risk squamous-cell carcinoma of the head and neck. N Engl J Med 2004;350:1937-1944.

48. Haddad R, O'Neill A, Rabinowits G, et al. Induction chemotherapy followed by concurrent chemoradiotherapy (sequential chemoradiotherapy) versus concurrent chemoradiotherapy alone in locally advanced head and neck cancer (PARADIGM): a randomised phase 3 trial. Lancet Oncol 2013;14:257-264.

49. Cohen EE, Karrison TG, Kocherginsky M, et al. Phase III randomized trial of induction chemotherapy in patients with $\mathrm{N}_{2}$ or $\mathrm{N}_{3}$ locally advanced head and neck cancer. J Clin Oncol 2014;32:2735-2743.

50. Geoffrois L, Martin L, De Raucourt D, et al. Induction chemotherapy followed by cetuximab radiotherapy is not superior to concurrent chemoradiotherapy for head and neck carcinomas: results of the GORTEC 2007-02 phase III randomized trial. J Clin Oncol 2018;36:3077-3083.

51. Zhong LP, Zhang CP, Ren GX, et al. Randomized phase III trial of induction chemotherapy with docetaxel, cisplatin, and fluorouracil followed by surgery versus up-front surgery in locally advanced resectable oral squamous cell carcinoma. J Clin Oncol 2013;31:744-751.

52. Bonner JA, Harari PM, Giralt J, et al. Radiotherapy plus cetuximab for squamous-cell carcinoma of the head and neck. N Engl J Med 2006;354:567-578.

53. Gillison ML, Trotti AM, Harris J, et al. Radiotherapy plus cetuximab or cisplatin in human papillomavirus-positive oropharyngeal cancer (NRG Oncology RTOG 1016): a randomised, multicentre, non-inferiority trial. Lancet 2019;393:40-50.

54. Mehanna H, Robinson M, Hartley A, et al. Radiotherapy plus cisplatin or cetuximab in low-risk human papillomavirus-positive oropharyngeal cancer (De-ESCALaTE HPV): an open-label randomised controlled phase 3 trial. Lancet 2019;393:51-60.

55. Tan HK, Giger R, Auperin A, Bourhis J, Janot F, Temam
S. Salvage surgery after concomitant chemoradiation in head and neck squamous cell carcinomas: stratification for postsalvage survival. Head Neck 2010;32:139-147.

56. Guo T, Qualliotine JR, Ha PK, et al. Surgical salvage improves overall survival for patients with HPV-positive and HPV-negative recurrent locoregional and distant metastatic oropharyngeal cancer. Cancer 2015;121:1977-1984.

57. Argiris A, Li S, Ghebremichael M, et al. Prognostic significance of human papillomavirus in recurrent or metastatic head and neck cancer: an analysis of Eastern Cooperative Oncology Group trials. Ann Oncol 2014;25:14101416.

58. Joseph AW, Guo T, Hur K, et al. Disease-free survival after salvage therapy for recurrent oropharyngeal squamous cell carcinoma. Head Neck 2016;38 Suppl 1:E1501-E1509.

59. Vermorken JB, Mesia R, Rivera F, et al. Platinum-based chemotherapy plus cetuximab in head and neck cancer. N Engl J Med 2008;359:1116-1127.

6o. Vermorken JB, Herbst RS, Leon X, Amellal N, Baselga J. Overview of the efficacy of cetuximab in recurrent and/ or metastatic squamous cell carcinoma of the head and neck in patients who previously failed platinum-based therapies. Cancer 2008;112:2710-2719.

61. Francisco LM, Salinas VH, Brown KE, et al. PD-L1 regulates the development, maintenance, and function of induced regulatory T cells. J Exp Med 2009;206:3015-3029.

62. Amarnath S, Mangus CW, Wang JC, et al. The PDL1-PD1 axis converts human $\mathrm{TH}$ c cells into regulatory $\mathrm{T}$ cells. Sci Transl Med 2011;3:111ra12o.

63. Bauml J, Seiwert TY, Pfister DG, et al. Pembrolizumab for platinum- and cetuximab-refractory head and neck cancer: results from a single-arm, phase II study. J Clin Oncol 2017;35:1542-1549.

64. Ferris RL, Blumenschein G Jr, Fayette J, et al. Nivolumab for recurrent squamous-cell carcinoma of the head and neck. N Engl J Med 2016;375:1856-1867.

65. Harrington KJ, Ferris RL, Blumenschein G Jr, et al. Nivolumab versus standard, single-agent therapy of investigator's choice in recurrent or metastatic squamous cell carcinoma of the head and neck (CheckMate 141): health-related quality-of-life results from a randomised, phase 3 trial. Lancet Oncol 2017;18:1104-1115.

66. Burtness B, Harrington KJ, Greil R, et al. Pembrolizum$\mathrm{ab}$ alone or with chemotherapy versus cetuximab with chemotherapy for recurrent or metastatic squamous cell carcinoma of the head and neck (KEYNOTE-O48): a ran- 
domised, open-label, phase 3 study. Lancet 2019;394:19151928.

67. Pestana RC, Becnel M, Rubin ML, et al. Response rates and survival to systemic therapy after immune checkpoint inhibitor failure in recurrent/metastatic head and neck squamous cell carcinoma. Oral Oncol 2020;101:104523.

68. Murphy BA, Deng J. Advances in supportive care for late effects of head and neck cancer. J Clin Oncol 2015;33:33143321.

69. Weber RS, Berkey BA, Forastiere A, et al. Outcome of salvage total laryngectomy following organ preservation therapy: the Radiation Therapy Oncology Group trial 9111. Arch Otolaryngol Head Neck Surg 2003;129:44-49.

70. van der Woerd B, Patel KB, Nichols AC, Fung K, Yoo J, MacNeil SD. Functional outcomes in early (T1/T2) supraglottic cancer: a systematic review. J Otolaryngol Head Neck Surg 2018;47:76.

71. Kumarasiri A, Liu C, Kamal M, et al. Changes in pharyngeal constrictor volumes during head and neck radiation therapy: implications for dose delivery. J Cancer Res Ther 2017;13:218-223.

72. Jacobi I, Navran A, van der Molen L, Heemsbergen WD, Hilgers FJ, van den Brekel MW. Radiation dose to the tongue and velopharynx predicts acoustic-articulatory changes after chemo-IMRT treatment for advanced head and neck cancer. Eur Arch Otorhinolaryngol 2016;273:487494.

73. Starmer HM, Tippett D, Webster K, et al. Swallowing outcomes in patients with oropharyngeal cancer undergoing organ-preservation treatment. Head Neck 2014;36:13921397.

74. Fung K, Lyden TH, Lee J, et al. Voice and swallowing outcomes of an organ-preservation trial for advanced laryngeal cancer. Int J Radiat Oncol Biol Phys 2005;63:13951399.

75. Kirsh E, Naunheim M, Holman A, Kammer R, Varvares M, Goldsmith T. Patient-reported versus physiologic swallowing outcomes in patients with head and neck cancer after chemoradiation. Laryngoscope 2019;129:2059-2064.

76. McClelland S 3rd, Andrews JZ, Chaudhry H, Teckie S, Goenka A. Prophylactic versus reactive gastrostomy tube placement in advanced head and neck cancer treated with definitive chemoradiotherapy: a systematic review. Oral Oncol 2018;87:77-81.

77. Lambert L, Fortin B, Soulieres D, et al. Organ preserva- tion with concurrent chemoradiation for advanced laryngeal cancer: are we succeeding? Int J Radiat Oncol Biol Phys 2010;76:398-402.

78. Grundmann O, Mitchell GC, Limesand KH. Sensitivity of salivary glands to radiation: from animal models to therapies. Version 2. J Dent Res 2009;88:894-903.

79. Hong CH, Napenas JJ, Hodgson BD, et al. A systematic review of dental disease in patients undergoing cancer therapy. Support Care Cancer 2010;18:1007-1021.

8o. Deng J, Jackson L, Epstein JB, Migliorati CA, Murphy BA. Dental demineralization and caries in patients with head and neck cancer. Oral Oncol 2015;51:824-831.

81. Epstein JB, Thariat J, Bensadoun RJ, et al. Oral complications of cancer and cancer therapy: from cancer treatment to survivorship. CA Cancer J Clin 2012;62:400-422.

82. Benson E, Li R, Eisele D, Fakhry C. The clinical impact of HPV tumor status upon head and neck squamous cell carcinomas. Oral Oncol 2014;50:565-574.

83. Rogers SN, El-Sheikha J, Lowe D. The development of a patients concerns inventory (PCI) to help reveal patients concerns in the head and neck clinic. Oral Oncol 2009;45:555-561.

84. Cohen EE, LaMonte SJ, Erb NL, et al. American Cancer Society head and neck cancer survivorship care guideline. CA Cancer J Clin 2016;66:203-239.

85. Pinto FR, Matos LL, Gumz Segundo W, Vanni CM, Rosa DS, Kanda JL. Tobacco and alcohol use after head and neck cancer treatment: influence of the type of oncological treatment employed. Rev Assoc Med Bras (1992) 2011;57:171-176.

86. Chen AM, Vazquez E, Courquin J, Donald PJ, Farwell DG. Tobacco use among long-term survivors of head and neck cancer treated with radiation therapy. Psychooncology 2014;23:190-194.

87. Gritz ER, Carmack CL, de Moor C, et al. First year after head and neck cancer: quality of life. J Clin Oncol 1999;17:352-360.

88. Funk GF, Karnell LH, Christensen AJ. Long-term health-related quality of life in survivors of head and neck cancer. Arch Otolaryngol Head Neck Surg 2012;138:123-133.

89. Chen AM, Daly ME, Vazquez E, et al. Depression among long-term survivors of head and neck cancer treated with radiation therapy. JAMA Otolaryngol Head Neck Surg 2013;139:885-889.

90. Mitchell AJ, Ferguson DW, Gill J, Paul J, Symonds P. Depression and anxiety in long-term cancer survivors com- 
pared with spouses and healthy controls: a systematic review and meta-analysis. Lancet Oncol 2013;14:721-732.

91. Shinn EH, Valentine A, Jethanandani A, et al. Depression and oropharynx cancer outcome. Psychosom Med 2016;78:38-48.

92. Marur S, Li S, Cmelak AJ, et al. E1308: phase II trial of induction chemotherapy followed by reduced-dose radiation and weekly cetuximab in patients with HPV-associated resectable squamous cell carcinoma of the oropharynx. ECOG-ACRIN Cancer Research Group. J Clin Oncol 2017;35:490-497.

93. Yom SS, Torres-Saavedra P, Caudell JJ, et al. NRG-HNooz: a randomized phase II trial for patients with p16-positive, non-smoking-associated, locoregionally advanced oropharyngeal cancer. Int J Radiat Oncol Biol Phys
2019;105:684-685.

94. Massarelli E, William W, Johnson F, et al. Combining immune checkpoint blockade and tumor-specific vaccine for patients with incurable human papillomavirus 16-related cancer: a phase 2 clinical trial. JAMA Oncol 2019;5:67-73.

95. Ho A, Brana I, Haddad R, et al. Abstract PRo8: preliminary results from a phase 2 trial of tipifarnib in squamous cell carcinomas (SCCs) with HRAS mutations. Mol Cancer Ther 2019;18(12 Suppl):PRo8.

96. Anderson CM, Lee CM, Saunders DP, et al. Phase IIb, randomized, double-blind trial of $\mathrm{GC}_{4419}$ versus placebo to reduce severe oral mucositis due to concurrent radiotherapy and cisplatin for head and neck cancer. J Clin Oncol 2019;37:3256-3265. 